Article

\title{
Optimal Charging Strategy Based on Model Predictive Control in Electric Vehicle Parking Lots Considering Voltage Stability
}

\author{
Beom-Ryeol Choi ${ }^{1}$, Won-Poong Lee ${ }^{2}$ and Dong-Jun Won ${ }^{2, *}$ \\ 1 Hyosung Corporation, 74, Simin-daero, Dongan-gu, Anyang-si, Gyeonggi-do 14080, Korea; \\ choico@hyosung.com \\ 2 Department of Electrical Engineering, Inha University, 100, Inha-ro, Michuhol-gu, Incheon 22212, Korea; \\ poong89@gmail.com \\ * Correspondence: djwon@inha.ac.kr; Tel.: +82-32-860-7404; Fax: +82-32-863-5822
}

Received: 19 June 2018; Accepted: 6 July 2018; Published: 11 July 2018

\begin{abstract}
Recently, electric vehicles (EVs) using energy storage have gained attention over conventional vehicles using fossil fuels owing to their advantages such as being eco-friendly and reducing the operation cost. In a power system, an EV, which operates through the energy stored in the battery, can be used as a type of load or energy source; hence, an optimal operation of EV clusters in power systems is being extensively studied. This paper proposes an optimal strategy for charging EVs in parking lots. This strategy is based on the model predictive control (MPC) framework due to the uncertainty of loads, renewable energy sources, and EVs, and considers the voltage stability of the distribution systems. EV chargers in the parking lot charge EVs to minimize the charging cost, which results in a sudden increase in charge load at a certain time. As a result, an excessive voltage drop may occur in the power system at that time. Therefore, we need to minimize the charging cost of EVs while preventing an excessive voltage drop in the power system. The parking lot is stochastically modeled to consider EV uncertainty under the MPC framework. In the MPC framework, the charging schedule of an EV charger in the parking lot is optimized by considering both voltage stability and charging cost minimization in real time. The charging constraints on voltage stability are updated through parameters that change in real time, and thus, errors caused by uncertainty can be reduced. Subsequently, this charging strategy is applied to multiple chargers through Monte Carlo simulation. The proposed charging strategy is verified based on MATLAB/Simulink.
\end{abstract}

Keywords: power system; electric vehicle; parking lot; model predictive control; voltage stability; Monte Carlo simulation

\section{Introduction}

As a new accord that was adopted in 2015 as a replacement for the Kyoto Protocol adopted in 1997, the Paris Climate Change Accord has led to the implementation of greenhouse gas reduction targets for all 195 participating countries. Starting with the Paris Climate Change Accord, renewable energy is spreading globally to become a competitive mainstream energy source for environmentally friendly development. In accordance with this global trend, recently, power systems have been undergoing a change from being central operation-based on a power plant using fossil fuels to employing a distributed operation, which mainly uses renewable energy and energy storage devices. In addition, there has been considerable research in various fields such as microgrids, energy storage systems, renewable energy, and electric vehicles (EVs) for operating power systems in a distributed manner. Of these, EVs are attracting maximum attention because they will be the most influential fields in the future as their penetration progresses. In addition, since EVs in power systems can be regarded 
as a sort of mobile battery, power system operators such as the microgrid need to carefully consider the uncertainties of EVs. Therefore, EVs have been studied by considering their uncertainty patterns. Specifically, issues such as how EVs are handled and how they affect distributed systems, such as microgrids and smart grids, are being researched.

Reference [1] proposes a model predictive control (MPC)-based method with the goal of minimizing the charging energy cost of the charging station when an EV is charged in it. The charging station performs charging control of the EVs by considering the electric price to exhibit the time-varying characteristic and the power reference change by providing auxiliary services to the distribution system operator. Reference [2] proposes a real-time algorithm that coordinates the charge of multiple plug-in electric vehicles to reduce stress that affects system stability and reliability. When coordinating the charge for purposes of supplying power to the $\mathrm{EV}$, and thereby, minimizing power loss, it uses voltage constraints, demand constraints over time, and the user's preferred charging time. Reference [3] describes how an aggregator in a system with a medium voltage level reprofiles the charging load of EVs. Moreover, a reference architecture for managing EVs in a cluster is proposed. Reference [4] describes in detail the impact that an EV can have when it increases in a low-voltage distribution grid. As a result, as the number of EVs increases, not only the electrical load but also the thermal load is increased, which can cause it to deviate from the normal operating range. This means that adjustments need to be made for charging several EVs simultaneously. Reference [5] suggests a controller that can handle the plug-and-play of charging of MPC-based EVs. This controller has the upper and lower limits of the voltage of the busbars of the power network as a constraint, and considers this constraint when charging an EV. This paper focuses on controller design and achieves a better control performance by reflecting a change in the connection state of EVs. Reference [6] suggests ways to reduce congestion in a distribution system by adjusting the charging of EVs by using the locational marginal pricing scheme. Reference [7] considers the uncertainty of charging EVs through the probability model and uses it for system operation and system planning. Reference [8] presents vehicle-to-parking and parking-to-vehicle schemes for EVs in each home and parking lot through a stochastic model and analyzes their effect on the power system. Reference [9] suggests a way to satisfy both the minimization of power generation cost and the system constraint by hierarchical charging control considering the uncertainty of EVs. Reference [10] presents charging control schemes for EVs considering transmission congestion. Reference [11] proposes a method to estimate the optimal capacity and location of EV parking lots in a distribution system through a stochastic approach. In Reference [12], the charging preference of the driver is considered through the utility function, and the charge scheduling of a plug-in hybrid electric vehicle is performed by adjusting the real-time charging price based on the charging preference. Reference [13] proposes an optimal charging strategy at the charging station by considering the navigation of the EV and the condition of the charging station, and applies a hierarchical game approach that maximizes the benefit of each charging station through competition between the charging stations. Reference [14] describes a strategy of minimizing the cost of the charging station by optimizing the charging operation in an EV parking lot by using two-stage approximate dynamic programming. In addition, strategies that maximize the benefits of aggregators and sub-components when participating in the market by considering aggregated load and distributed power and EV together are proposed in [15] and a stability management method of power systems based on equilibrium price through market-based operation of the aggregator is studied.

Besides the abovementioned studies, various studies are ongoing as EVs become more influential, and there are some features that are derived from these EV studies. First, EVs can be considered as moving batteries, so there is uncertainty as to the usage patterns of users. Second, if multiple EVs are connected to a power system, they have a significant impact on system stability. Finally, many EVs can be aggregated in places such as charging stations or parking lots, which can be managed through an aggregator, and the management of costs such as market participation or charge fees is essential.

For these reasons, this paper proposes an optimal charging strategy of EVs in a parking lot through an aggregator. To account for the uncertainty of EVs, a stochastic approach is applied to the 
pattern of EVs entering the parking lot and an MPC-based framework is used to reflect the change in the state of EVs and electric tariff in the charging strategy. In addition, voltage constraints based on the voltage sensitivity of the system buses are generated to account for voltage drops that can occur when EVs perform charging in the distribution grid, which is reflected in the charging strategy. Therefore, the proposed charging strategy can achieve optimization of the charging rate while considering the voltage stability of the system.

In summary, this paper makes the following contribution.

(1) It is a charging station operation strategy that considers charging rate optimization of EVs in a parking lot and voltage stability of the power system. This strategy can be applied to an aggregator that manages parking lots or charging stations where EVs are assembled. This strategy can be used to prevent the bus voltage drop in the grid due to EV charging during the day, and EV customers can be provided with a minimum charging rate.

(2) A relatively simple voltage constraint based on voltage sensitivity is used to prevent voltage drop caused by concentrated charging of EVs. This constraint can be updated in real time and applied only when needed. Due to the uncertainty of EVs, it is difficult to specify the time at which the voltage drop will occur, and for the aggregator, there is no guarantee that the state of the power system will always be verified when the operating strategy is established. When this constraint is used, basically, the operating strategy is set up through charging rate optimization, and can be applied only to the section where the voltage drop is expected to occur, so that the influence of concentrated charging of EVs on the system can be minimized.

(3) Finally, the uncertainty of residual state-of-charge (SOC) and entry time of EVs entering the parking lot is substantial compared to the deterministic approach, because it is considered as a stochastic approach. In addition, since the voltage constraint is updated in real time based on the MPC framework, it is possible to cope with the variation in the condition inside the charging station.

The rest of this paper is organized as follows. Section 2 presents the basic model for the proposed strategy, describing the stochastic model, MPC-based framework, and Monte Carlo simulation for the EV parking lot used. Section 3 describes an optimal charging strategy for EV parking lots. Section 3.1 describes a strategy that is basically aimed at minimizing the charging rate. Section 3.2 describes an optimal charging strategy that accounts for the voltage variations in the power system. Section 4 presents the simulation results for verification of the proposed strategy. Finally, Section 5 concludes the study.

\section{The Basic Model Configuration for the Proposed Strategy}

\subsection{Stochastic Modeling of EV Parking Lot}

Since an EV is an uncontrollable load for the aggregator, the parking lots for the following three items are stochastically constructed and modeled to generate new probabilities every hour: (1) the time when an EV enters the parking lot; (2) the time when an EV leaves the parking lot; and (3) the remaining SOC of the EV battery when entering. If the charger is already being used by the EVs determined by the probability of entry and exit, then the next EV cannot use the charger. A charging index is defined to account for this situation: if the charging index is activated (for example, if it is considered a binary variable), it means that there is an EV currently being charged in the charger. Figure 1 shows a schematic of the daily charging schedule according to stochastic EV entry/exit from the charger perspective. 


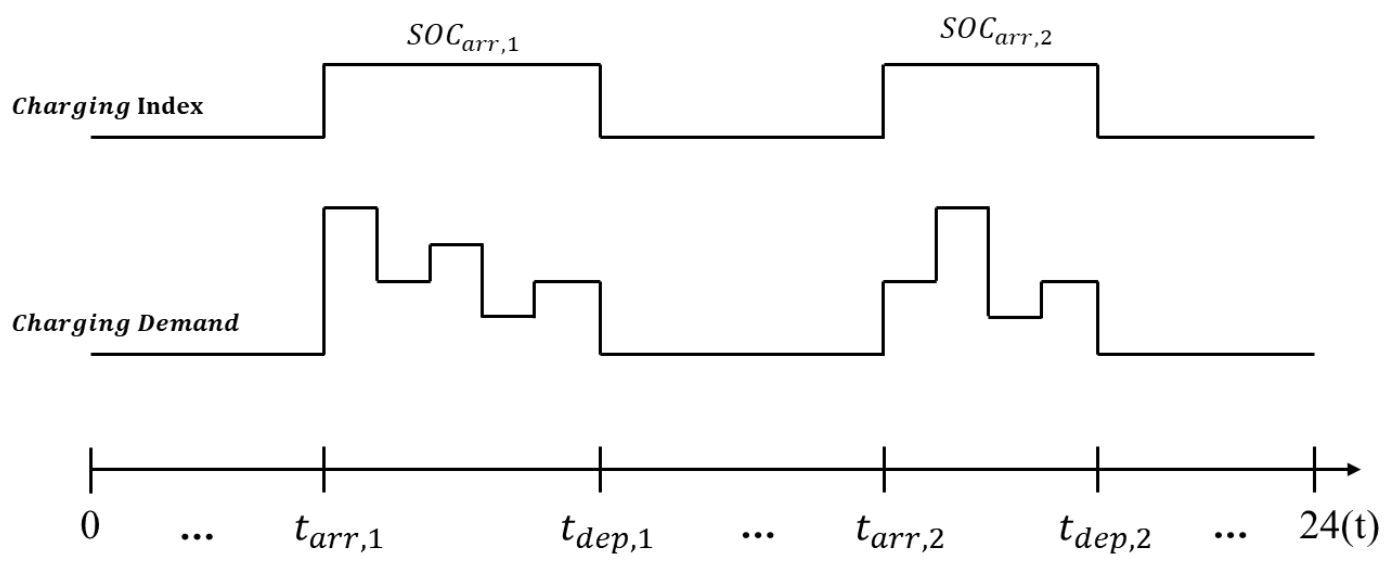

Figure 1. Probability Generation Modeling Schematic.

$t_{a r r, 1}, t_{d e p, 1}$, and $S O C_{a r r, 1}$ indicate the entry and departure times of the EV that first arrived at the charger and the remaining SOC, respectively, which are determined stochastically. Based on the probability of entry time, the EV is determined to be charged at that time and the proposed strategy is applied according to the remaining SOC and time of departure. In Figure 1, the charging index is activated from 0 to 1 when EV charging is determined at $t_{a r r, 1}$. The charging demand indicates the result of applying the proposed strategy.

As mentioned above, the times of entry and departure of the vehicle and the remaining SOC are considered as stochastic variables. Since all three variables are random, some factors need to be considered. First, if the departure time is earlier than the entry time, it should be excluded, because it is practically meaningless. Second, while the EV is in the parking lot, the chargeable amount must not exceed the capacity of the EV battery. Third, the amount to charge must be achievable within the time in the parking lot. If the user gives the SOC constraint form for the required charge amount considering the second and third conditions, the physical constraint can be clearly generated, but if not, the chargeable amount is also determined stochastically. If the condition is not met, it is excluded from the stochastic results. Figure 1 is an example applied to each charger, and since there are many EV chargers in the parking lot, the charging strategy of the parking lot during the day can be established by the result of the probability per charger of the parking lot. Since each charger has its own independent random variables, in this paper, a charging schedule for several chargers is configured, which are summed to be considered in the parking lot. The EV battery capacity differs depending on the EV model; however, in this paper, the capacity is unified through one EV model.

$$
\begin{gathered}
t_{\text {arr }, n}<t_{\text {dep }, n} \\
t_{\text {dep }, n}-t_{\text {arr }, n}=t_{\text {parking }, n} \\
E_{\text {demand }, n}=\left(S O C_{\text {pref }, n}-S O C_{\text {arr }, n}\right) \times C_{\text {bat }, n} \\
E_{\text {demand }, n} \leq t_{\text {parking }, n} \times \eta_{\text {charger }, n} P_{\text {charger }, n}^{\max } \\
0<S O C_{\text {arr }, n} \leq 1
\end{gathered}
$$

Equations (1) and (2) indicate the constraints on the times at which the $n$-th EV arrives and leaves the parking lot. These constraints are used to generate probabilities for EVs. Equation (1) is a condition according to which the time to arrive at the parking lot, $t_{\text {arr }, n}$, must occur before the time to leave the parking lot, $t_{d e p, n}$, and Equation (2) is a condition according to which the difference between the two points is the time when the EV is parked. Equation (3) describes the condition for calculating the charging demand of the $n$-th EV. SOC arr, $n$ is the remaining SOC when the $n$-th EV arrives at the parking lot, $S O C_{p r e f, n}$ is the required SOC to be input, and $C_{b a t}$ is the battery capacity. The $S O C_{a r r, n}$ in Equation (3) 
is determined stochastically. Equation (4) implies that the charge requirement of the EV should be equal to or less than the maximum chargeable charge during the parked time. $\eta_{\text {charger }, n}$ indicates the charging efficiency of the charger, and $P_{c h a r g e r, n}^{\max }$ indicates the maximum output of the charger. Since $t_{a r r, n}, t_{d e p, n}$, and $S O C_{a r r, n}$ are determined stochastically, it cannot occur if the required amount of charge cannot be satisfied during the parking time, and thus, this case is excluded in Equation (4). Equation (5) represents the condition for the remaining SOC of the EV arriving at the parking lot.

\subsection{MPC Framework}

MPC is a control algorithm that finds an optimal control signal that minimizes the objective function defined by the user by considering the constraints based on the mathematical model [16]. In MPC, the state variable or output is predicted using the model and optimization is performed using an appropriate constraint and cost function over the prediction time horizon. MPC sets the prediction horizon, which is a prediction interval for the future, and optimizes the predicted value to minimize errors in the interval. Only the first control input value among the optimization values is used as the control input of the current time. These predictions are optimal control input values that minimize the cost function and satisfy the given constraints. In the next time step, the same procedure as above is repeatedly performed and an optimal control input is applied to the corresponding model, so that optimized control can always be achieved. The basic system structure of MPC is shown in Figure 2.

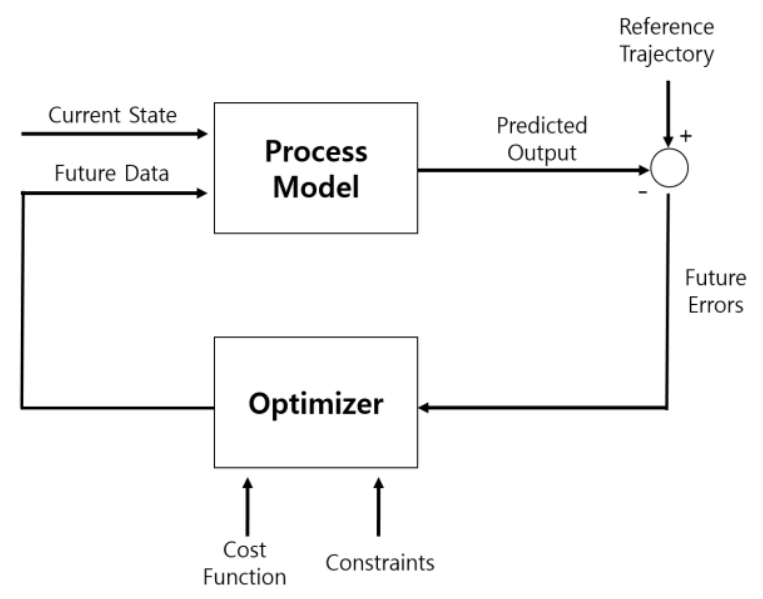

Figure 2. The basic structure of MPC (Model Predictive Control).

Since MPC is a control technique, only its framework is used in this study to develop the charging strategy. When the optimal charging strategy is designed, the error between the optimum points can be substantially reduced by updating the parameters and constraints that change in real time. In this paper, the constraint that is changed in the MPC framework is the bus voltage limit of the power system. Since the charging of the EV is always performed in real time, it is necessary to set the charging limit in advance by predicting the future charging load and voltage limit. Therefore, the charging limit is set at the time when the voltage drop is expected through the MPC, and is prevented through the charging constraints at the corresponding charging time.

\subsection{Monte Carlo Simulation}

Monte Carlo simulation is a simulation method for probabilistically obtaining the value of a desired equation by using randomly extracted numbers. It is also called the simulated sampling technique because it randomly selects a number from a probability distribution for use in one simulation run. The task of estimating the probability distribution of the target function by deriving the pattern of the unit variable through the generated random number is the core of the Monte Carlo simulation method. 
In this study, the EV parking lot is designed based on the stochastic model considering the moving pattern of the EVs. The probabilistic model for the EV charger can be extended based on Monte Carlo simulation. That is, the total number of repetitions of the Monte Carlo simulation is the number of chargers that form the entire parking lot, and the probability of being used in the charger is independent of each repetition of the probability distribution associated with the EV. It is possible to integrate all charging loads of the EV generated in each charger and regard it as the total load in the parking lot.

\section{Optimal Charging Strategy of EV Parking Lot}

This section describes a strategy that optimally charges the EV while considering the voltage limit of the power system and keeping the charge cost to a minimum when EVs are charging in the parking lot.

An EV parking lot with this structure, which has several EV chargers, and an aggregator that includes residential loads, are designed. Overall, the optimal charging strategy comprises a two-level hierarchical structure. First, the optimum charging simulation is performed considering only the charging rate. Here, a reference to the optimal charge schedule for $24 \mathrm{~h}$ is determined, and the determined value can be applied as an initial value for the parking lot operation. Second, the aggregator calculates the corresponding low voltage limit that can mitigate the voltage drop considering the voltage stability of the entire power system. Based on the calculated voltage limit, each parking lot operator reconfigures the charging schedule. The second step is performed in the MPC framework when the current operational state, which means the bus voltage, changes. When charging occurs in real time, depending on the entry of probabilistic EVs and the variability of local loads, the constraints on voltage need to change every hour. The aggregator checks the voltage limit according to the current load condition and updates the low voltage limit every hour if the current low voltage limit deviates from the minimum voltage limit. That is, if the voltage drop is less than the threshold value defined by the operator, the second step is not performed, whereas if the voltage deviates from the threshold value due to a severe voltage drop, the second step is applied. After the updated voltage constraint is reflected, the EVs are charged to account for charging constraints on the voltage, thereby preventing an excessive voltage drop. Figure 3 shows the structure of the EV parking lot, which forms the basis of the strategy proposed in this paper.

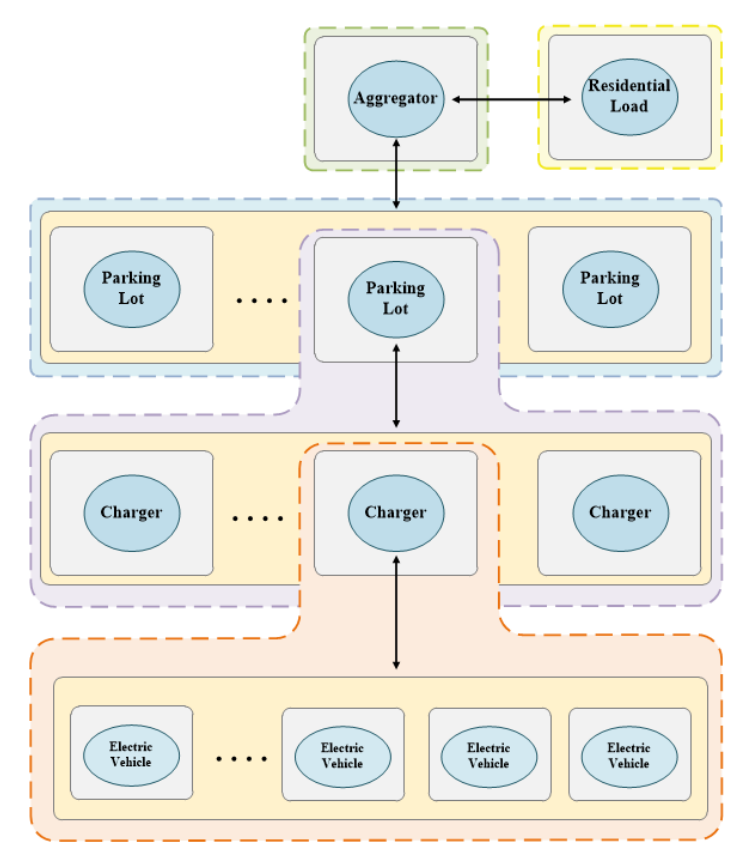

Figure 3. The proposed hierarchical structure of EV parking lots. 


\subsection{Optimal Charging Strategy Considering Charging Rate}

Based on the probabilistic modeling of the EV parking lot, the first optimal charging schedule is derived. Depending on the EV charging rate, the charging is performed continuously during the parking time based on the following three probabilities: the probability of the EV entering the parking lot, the probability of the EV leaving the parking lot, and the probability of SOC remaining upon entry. The charge rate for EVs is set as a time-of-use (TOU) charge. Since minimizing the charging rate is considered in the optimal charging strategy, the optimization proceeds so that the charging rate is minimized while achieving full charge to meet the conditions during the parking time. The amount of charge for each time is determined according to the constraint that does not exceed a maximum value determined according to the efficiency of the charger.

$$
\begin{gathered}
\min \sum_{t=t_{\text {arr }, n}}^{t_{\text {dep }, n}}\left(C_{T O U, t} \times P_{\text {demand }, n, t}\right) \\
\sum_{t=t_{\text {ar }, n}, n}^{t_{\text {dep }, n}} P_{\text {demand }, n, t} \Delta t=E_{\text {demand }, n} \\
S O C_{n, t+1}=S O C_{n, t}+\frac{\eta_{\mathrm{EV}, n} P_{\text {demand }, t, n}}{C_{\text {bat }}} \Delta t \\
0 \leq P_{\text {demand }, n, t} \leq \eta_{\text {charger }, n} P_{\text {charger }, n}^{\max } \\
0 \leq S O C_{n, t} \leq S O C_{\text {pref }, n}
\end{gathered}
$$

Equation (6) represents the objective function for minimizing the charging rate. Here, $P_{\text {demand }, n, t}$ and $C_{\mathrm{TOU}, t}$ denote the EV charging amount and charging rate for time $t$, respectively. Equations (7)-(10) represent constraints on the optimization problem. Equation (7) implies that the stochastically determined charging demand equals the sum of the charger output results during the parked time. Equation (8) is the constraint on the SOC update of the EV during the parking time $t_{\text {parking, } n}$, Equation (9) indicates the upper and lower limits of $P_{\text {demand, } n, t}$, and Equation (10) is the SOC upper and lower limits of the EV battery during the parking time. These equations identify the optimal charging amount for each time step so that the total cost is at its minimum during the parking time. Because the optimization problem is solved for one charger, the same problem is applied to multiple chargers in the parking lot through the Monte Carlo simulation. Therefore, the number of simulations is specified as the total number of chargers in a single parking lot. As a result, when the charging loads derived from the results of all simulations are summed up, the load resulting from the charging of the EVs in a single parking lot can be calculated. Figure 4 is a flowchart of the optimal charging strategy considering the electric charging rate.

The charging strategy that considers the charging rate is only optimized to minimize the total charging cost based on the charging rate of the EV. Therefore, the charging load can be concentrated at a time when the charging rate is low, which may cause an increase in the load in the area and a serious voltage drop. Minimization of the charge rate has been achieved, but since voltage drops have occurred, voltage stability may be adversely affected, and so a charging strategy with voltage limitations is needed. In the next section, the charging strategy is discussed, which can achieve a minimum charge rate while preventing voltage drop. 


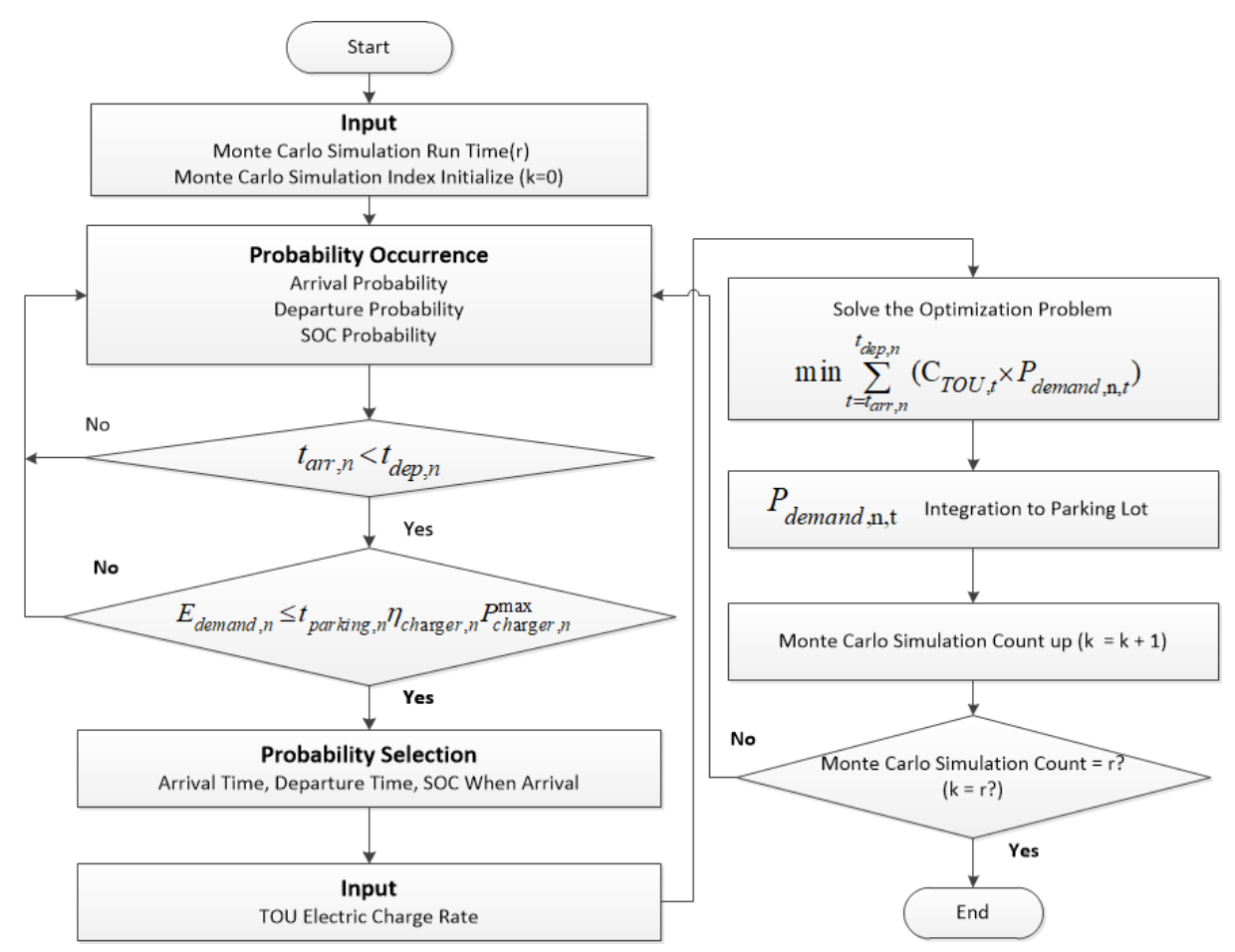

Figure 4. Flow-Chart of Optimal Charging Strategy Considering Charging Rate.

\subsection{Optimal Charging Strategy Considering Voltage Stability Based on MPC Framework}

To consider the voltage limit, the concept of voltage sensitivity using the relation between the bus voltage and active power according to the local loads in the power system is used [17]. The voltage variation effect is a computed result of the effect of load variation of different buses on the voltage change in a particular bus using voltage sensitivity. Thus, the effect of EV charging with respect to the voltage on the node and other nodes can be calculated as a voltage variation effect. Since the proposed voltage variation effect is not considered for line impedance, it may be different from the actual voltage drop or voltage variation. However, when the power system is designed, the basic analysis is performed and the voltage sensitivity is set according to the analysis result, so that the voltage variability for each bus does not change significantly if the system topology is not changed. Thus, using the voltage variation effect, the estimated active power value for the voltage deviation can be calculated when the voltage deviation exceeds a certain predefined level.

$$
\begin{gathered}
u_{i j}=\left(V_{r e f}-V_{l b, j}\right) / \Delta P_{\max , i}=\frac{\Delta V_{\max , j}}{\Delta P_{\max , i}} \\
U=\left[\begin{array}{cccc}
u_{11} & u_{12} & \cdots & u_{1 n} \\
u_{21} & u_{22} & \cdots & u_{2 n} \\
\vdots & \vdots & \ddots & \vdots \\
u_{n 1} & u_{n 2} & \cdots & u_{n n}
\end{array}\right]
\end{gathered}
$$

Equation (11) defines the voltage sensitivity, which indicates the relation between the change in the active power at bus $i$ and the variation in voltage at bus $j$. This can be used to calculate the variable active power according to the maximum allowable voltage. Since the voltage level of the power system and the allowable bus voltage value depend on the grid, sensitivity is defined by the voltage and power variation values $\left(\Delta V_{\max , j} / \Delta P_{\max , i}\right)$ in order to take into account the voltage stability, and the constraint on the voltage is to reduce this variation. In Equation (11), the reference voltage refers to the voltage at the point of common coupling and can be set to a slack voltage value. In addition, $\Delta V_{l b, j}$ 
indicates the variable lower limit of the voltage of the $j$-th bus and $\Delta P_{\max , i}$ indicates the maximum value of the allowable active power variation of the $i$-th bus. A matrix of defined sensitivity indices is given in Equation (12).

$$
\begin{gathered}
\Delta V_{e f f, j, t}^{\text {load }}=\sum_{i=1}^{N} u_{i j}\left(P_{i, t}^{\text {load }}+\Delta P_{i, t}^{\text {load }}\right) \\
\Delta V_{e f f, j, t}^{E V}=\sum_{i=1}^{N} u_{i j} \Delta P_{i, t}^{E V} \\
\Delta V_{e f f, j, t}^{\text {total }}=\Delta V_{e f f, j, t}^{\text {load }}+\Delta V_{e f f, j, t}^{E V}
\end{gathered}
$$

Using the previously defined sensitivity index matrix, the aggregator finds the voltage variation of bus $j$ according to the variability of loads on each bus, as shown in Equation (13). It shows the current state of the voltage variation due to the predicted load and the variation of the load. Similarly, Equation (14) defines additional voltage variations depending on the charging load of EVs. As a result, Equation (15) shows the total voltage variation at the present time when the load and load variability and the charging power of the EVs are all considered.

$$
\begin{gathered}
\Delta V_{e f f, j, t}^{\text {total }}>L V_{\lim } \Delta V_{\max , j} \\
\Delta V_{e f f, j, t}^{\text {reduced }}=\Delta V_{e f f, j, t}^{\text {total }}-L V_{\lim } \Delta V_{\max , j} \\
\Delta P_{\text {reduced }, i, t}^{E V}=\frac{\Delta V_{e f f, j, t}^{\text {reduced }}}{u_{i j}} \\
0 \leq P_{\text {demand }, n, i, t} \leq\left(P_{\text {demand }, n, i, t}^{\text {prev }}-\Delta P_{\text {reduced }, i, t}^{E V}\right)
\end{gathered}
$$

Equation (16) shows the conditions when additional constraints are generated to reduce voltage variations. Additional constraints are generated when the total voltage variation is above a certain level for the maximum allowable variation. If Equation (16) is not satisfied, no additional constraint is generated. $L V_{\lim }$ is a parameter determined by the operator as a specific level of voltage variation.

Equation (17) shows the voltage variations, $\Delta V_{e f f, j, t}^{r e d u c e}$, that need to be reduced. In Equation (18), the effective power value that is substantially reduced is derived from the voltage variation value calculated in Equation (17) and the previously defined voltage sensitivity index value. This value is applied for the total EV charging loads connected to bus $i$ for the corresponding time $t$, bus $j$. Equation (19) shows the constraints that should be additionally considered when the optimization problem for the EV charge schedule is re-solved. Equation (19) is the upper limit condition, $P_{\text {demand }, n, i, t}^{\text {pre }}$ is the EV charging load value determined in the previous problem, and $P_{\text {demand }, n, i, t}$ is the decision variable in the optimization problem. Since this optimization problem is solved within the MPC framework, Equation (19) may not apply if the state of the power system changes every hour. That is, when the value of the voltage variation is recovered within the predetermined range, the corresponding constraint is again excluded, the rescheduling is performed, and the charging rate is minimized. Figure 5 shows a flowchart of the optimal charging strategy considering voltage variation in the MPC-based framework. 


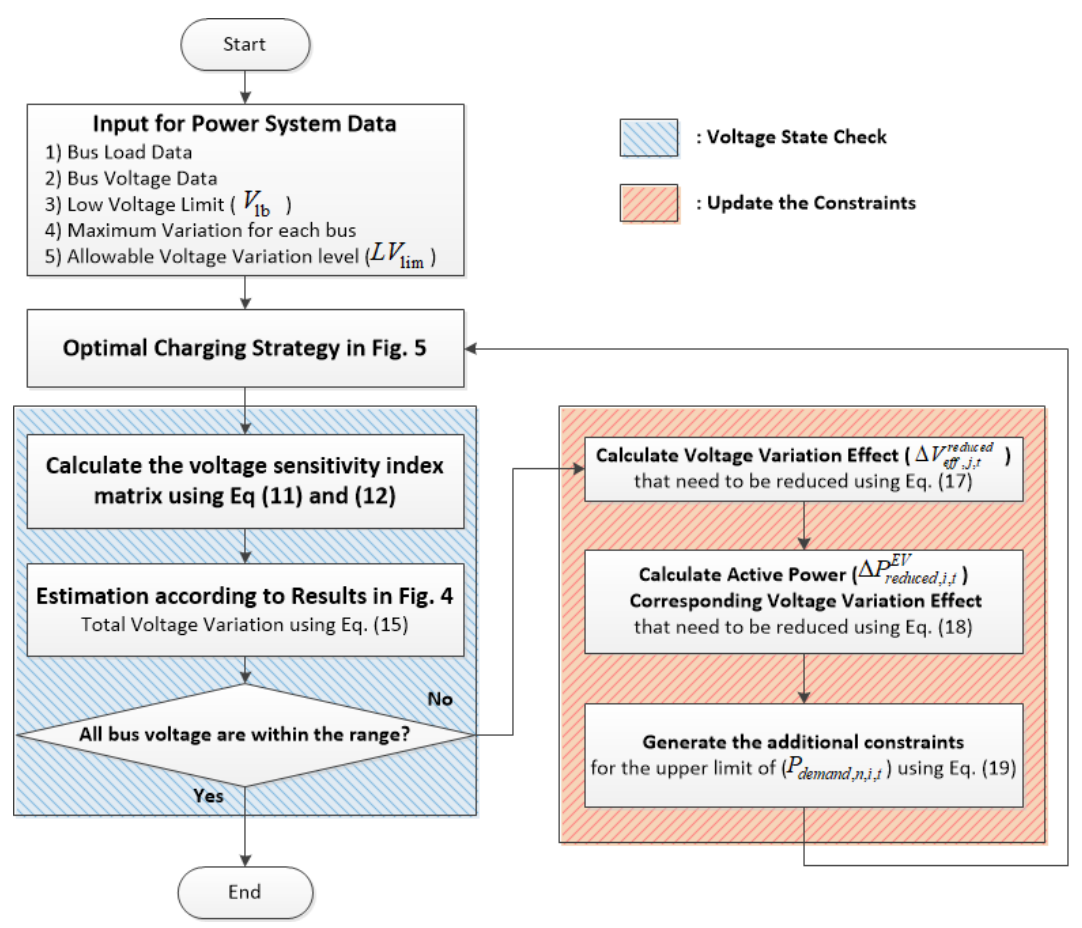

Figure 5. Flow-Chart of Optimal Charging Strategy Considering MPC-based Voltage Stability.

\section{Case Study for the Verification of the Proposed Strategy}

In this section, strategy verification is performed on the IEEE 15 bus system. In this study, a simulation model of the system, including an EV parking lot, is constructed by referring to the IEEE 15 bus system and three EV parking lots are set up. Figure 6 shows the IEEE 15 bus system including the EV parking lot structure used in the simulation. The arrival and departure probabilities in each EV parking lot are set differently and the simulation is performed under the same conditions as those for the residual SOC and TOU for charging. The parameters of the IEEE 15 bus system are shown in Tables 1 and 2. The EV is set to the Nissan Leaf model and the battery capacity is $24 \mathrm{kWh}$. The charger is set to a $6.6 \mathrm{~kW}$ charge model with $3.3 / 6.6 \mathrm{~kW}$ as a slow charge model, and the time step in the optimization problem is set to $1 \mathrm{~h}$.

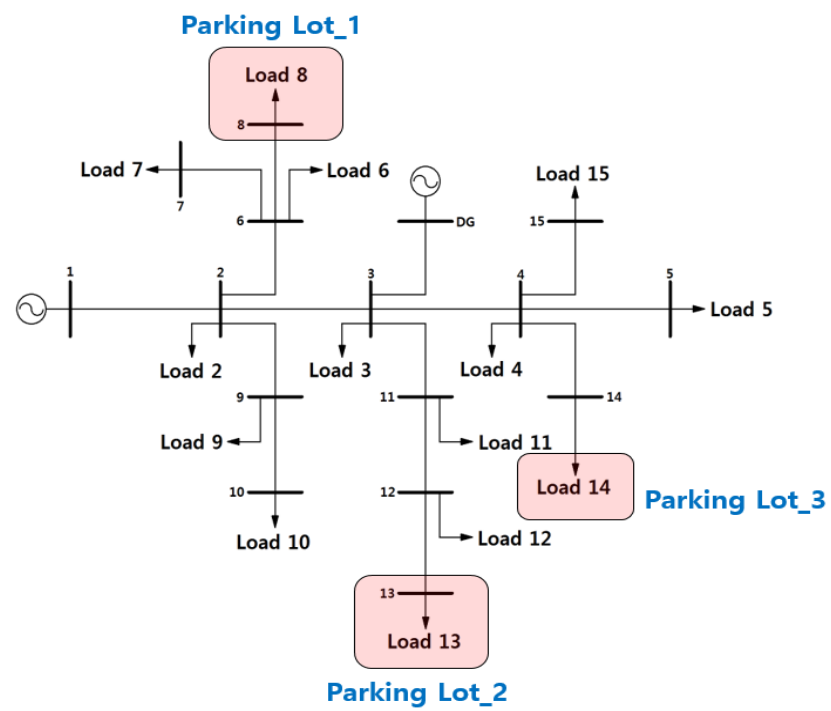

Figure 6. A schematic diagram of the IEEE 15 bus system with three EV parking lots. 
Table 1. IEEE 15 Bus System Load Data.

\begin{tabular}{cccc}
\hline Bus & PL (kW) & Bus & PL (kW) \\
\hline 2 & 44.1 & 9 & 70 \\
3 & 70 & 10 & 44.1 \\
4 & 140 & 11 & 70 \\
5 & 44.1 & 12 & 44.1 \\
6 & 140 & 13 & 140 \\
7 & 70 & 14 & 140 \\
8 & 140 & 15 & 70 \\
\hline
\end{tabular}

Table 2. IEEE 15 Bus System Line Data.

\begin{tabular}{ccccc}
\hline From & To & $\mathbf{R}(\boldsymbol{\Omega})$ & $\mathbf{X}(\boldsymbol{\Omega})$ & $\mathbf{L}(\boldsymbol{\Omega})$ \\
\hline 1 & 2 & 1.35309 & 1.32349 & 0.0035107 \\
2 & 3 & 1.17024 & 1.14464 & 0.0030363 \\
3 & 4 & 0.84111 & 0.82271 & 0.0021823 \\
4 & 5 & 1.53248 & 1.0276 & 0.0027258 \\
2 & 9 & 2.01317 & 1.3579 & 0.0036019 \\
9 & 10 & 1.68671 & 1.1377 & 0.0030178 \\
2 & 6 & 2.55727 & 1.7249 & 0.0045754 \\
6 & 7 & 1.0082 & 0.734 & 0.0019470 \\
6 & 8 & 1.25143 & 0.8441 & 0.0022390 \\
3 & 11 & 1.79533 & 1.2111 & 0.0032125 \\
11 & 12 & 2.44847 & 1.6515 & 0.0043807 \\
12 & 13 & 2.01317 & 1.3579 & 0.0036019 \\
4 & 14 & 2.23081 & 1.5049 & 0.0039913 \\
4 & 15 & 1.19702 & 0.8074 & 0.0021417 \\
\hline
\end{tabular}

Figure 7 shows the probability of EV entry in three EV parking lots, and Figure 8 shows the probability of EVs departing from the parking lots. The arrival and departure probabilities of each parking lot are set with reference to [14,15]. As the probabilities of arrival and departure vary, the charging load required for each parking lot varies. Although the arrival and departure probabilities of EVs in each parking lot are different, the probability of the remaining SOC and electric price are simulated using the same probability. The SOC remaining in the EV battery when the EV enters the parking lot is set by referring to [14]. Figure 9 shows the probability values for the percentage of remaining SOCs. The TOU electricity price for EV charging is set to the low voltage price in the summer of the Korea Electric Power Corporation (KEPCO) EV charging price list. Figure 10 shows the KEPCO electric prices for EV charging used for algorithm verification. The proposed strategy is performed using MATLAB, and the IEEE 15 bus system is implemented using MATLAB/Simulink using a computer with an Intel Core i7-6800K $3.4 \mathrm{GHz}$ CPU and $16 \mathrm{~GB}$ memory.

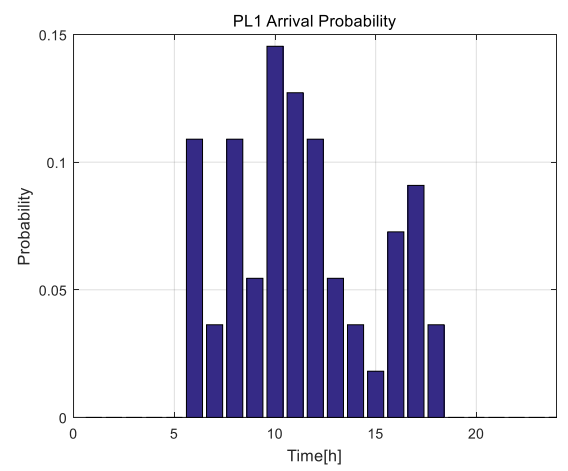

(a)

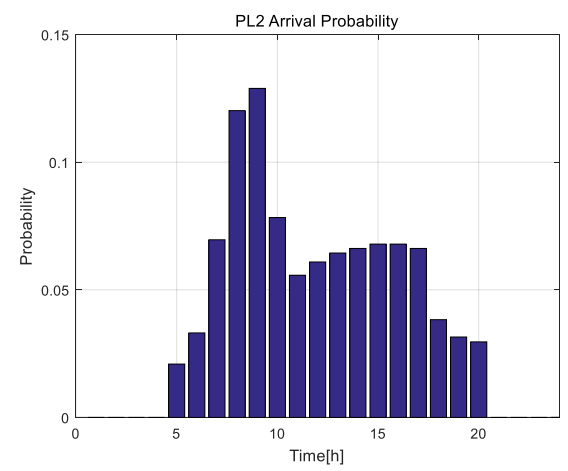

(b)

Figure 7. Cont. 


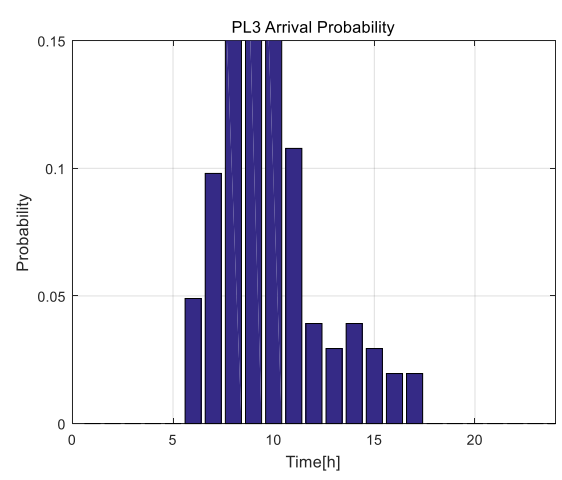

(c)

Figure 7. The Probability of Arrival at the Parking Lot (a) PL1, (b) PL2, (c) PL3.

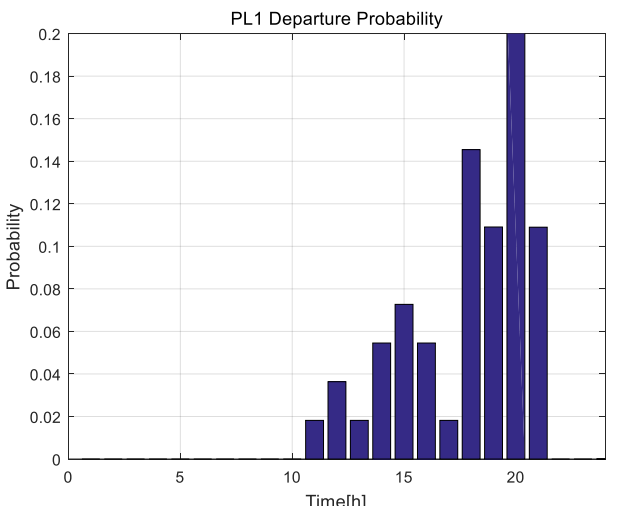

(a)

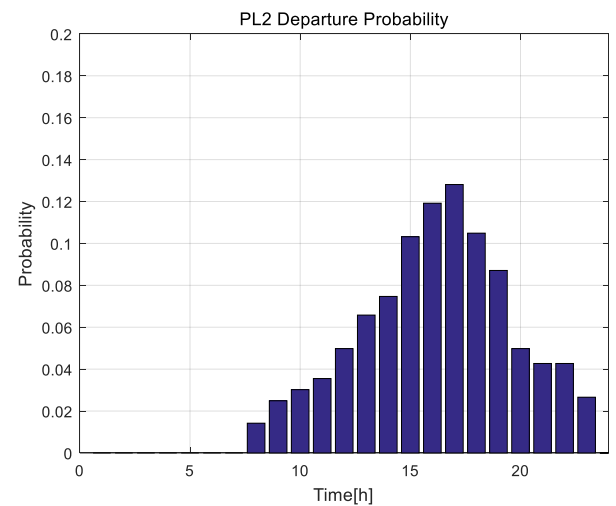

(b)

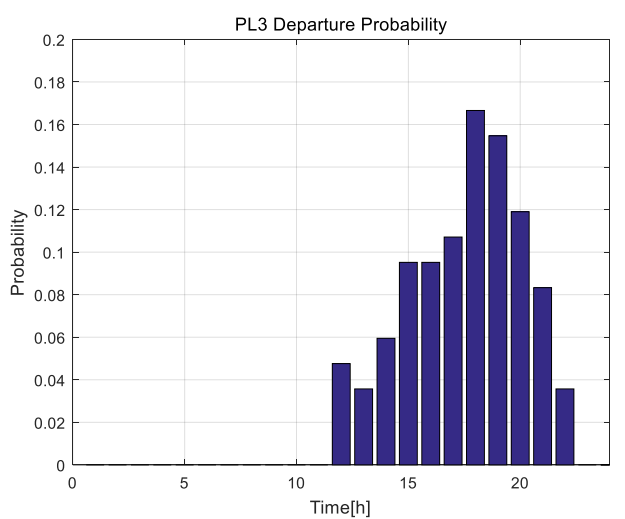

(c)

Figure 8. The Probability of Departure from the Parking Lot (a) PL1, (b) PL2, (c) PL3. 


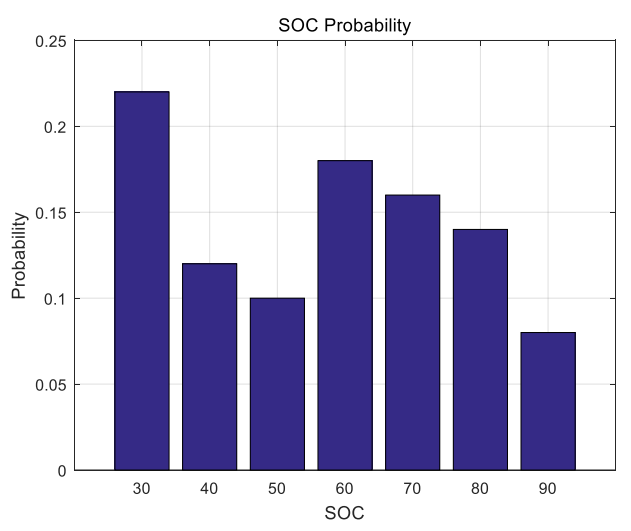

Figure 9. SOC Remaining Probability.

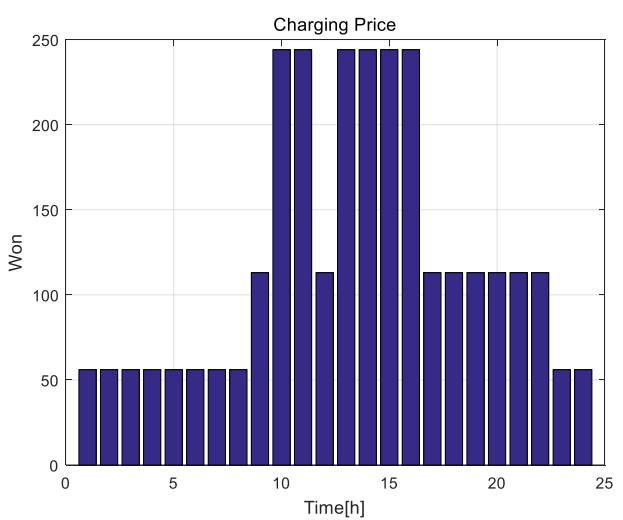

Figure 10. TOU Electricity Charging Rate.

Figures 11 and 12 show the total charging load of 30 chargers in each parking lot and the voltage drop at the bus to which the parking lot is connected, respectively. The number of chargers is set considering the existing load data of the IEEE 15 bus system, as shown in Table 1. Figure 11 shows the concentration of EVs charging from 6:00 to 8:00 in all parking lots because the EVs are optimally charged to minimize the total charging cost considering only the TOU electric price, and TOU is low at that time. On the other hand, the EVs hardly charge at 9:00 to 11:00 and 13:00 to 16:00 with high TOU.

This is considered to affect the voltage stability of the power system when a voltage drop occurs when the voltage variation exceeds $10 \%$. In this paper, when the maximum variability in the voltage is set to $10 \%$ and the voltage of the bus connected to the parking lot is lower than $0.9[\mathrm{pu}]$, the constraint considering the bus voltage is set to be generated. As a result, according to the TOU, the charge of the EVs is concentrated at a certain time (6:00-8:00) and the bus voltage to which each parking lot is connected is confirmed to drop below 0.9 [pu] at 7:00 to 8:00.

Figures 13 and 14 show the total charging load results and bus voltage where each parking lot is connected when additional constraints for the voltage drop are considered in the MPC-based framework. Each operator of the parking lot performs optimization through the receding horizon in the MPC framework, and then, the aggregator checks the bus voltage and updates the constraints for the charging power considering the voltage variation in real time. 


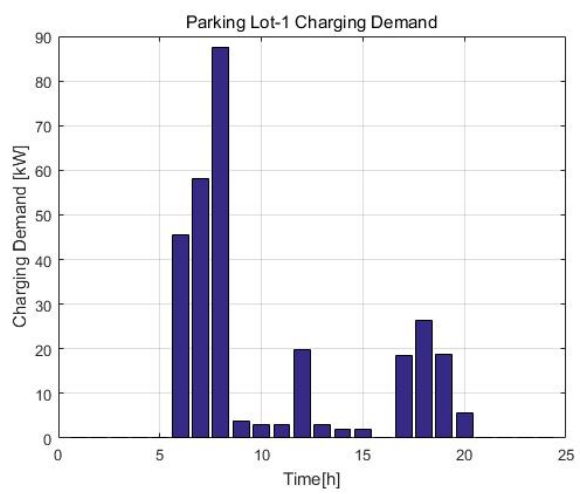

(a)

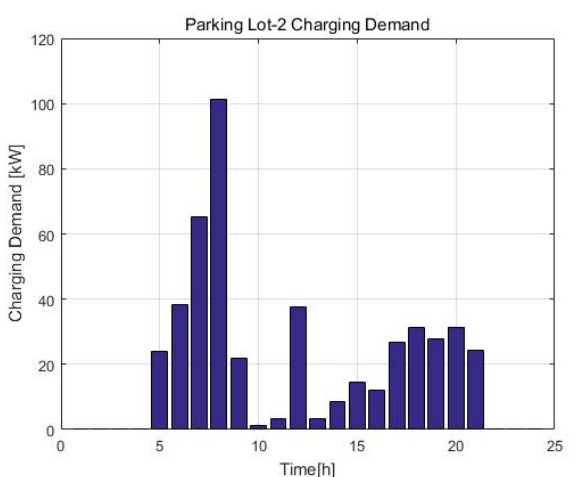

(b)

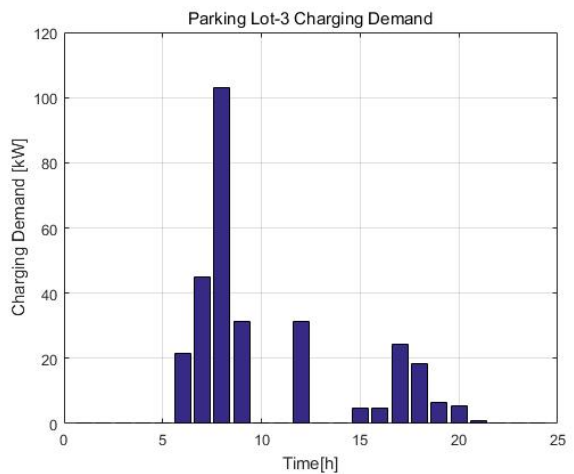

(c)

Figure 11. Total Charging Load of Parking Lot (a) PL1, (b) PL2, (c) PL3.

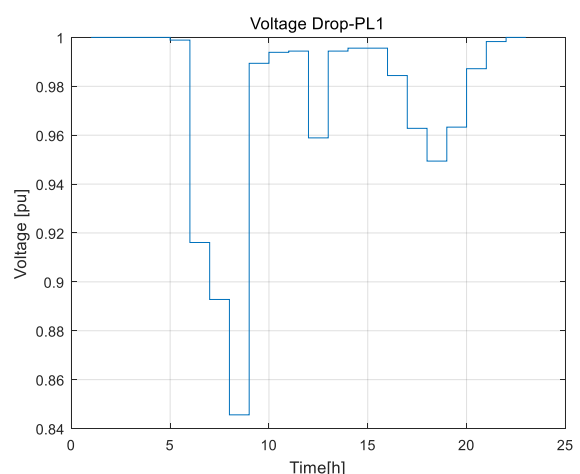

(a)

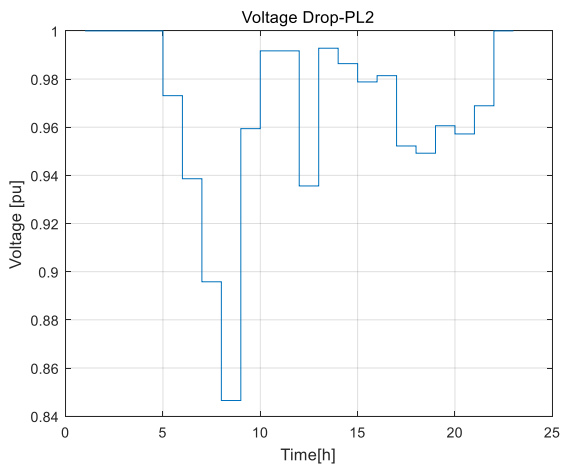

(b)



(c)

Figure 12. Voltage Variation in each Parking Lot (a) PL1, (b) PL2, (c) PL3. 


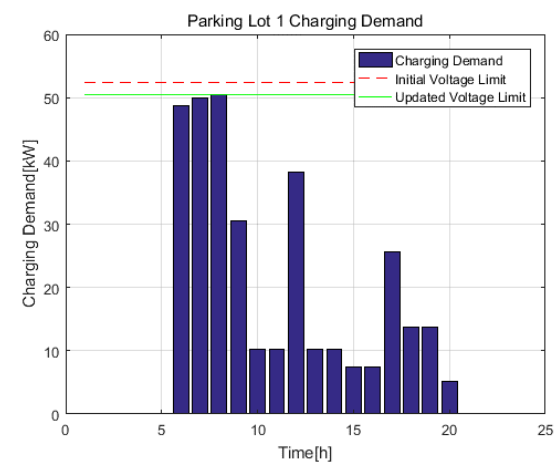

(a)

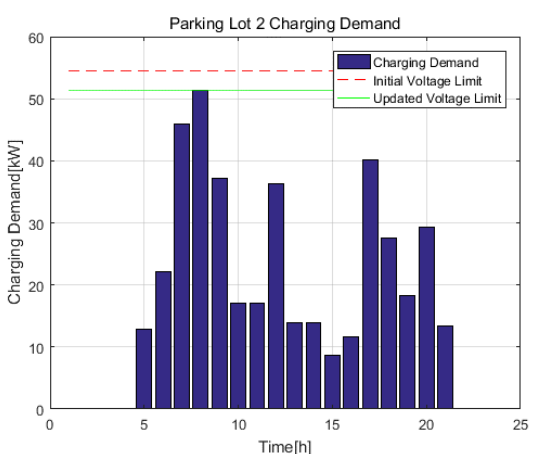

(b)

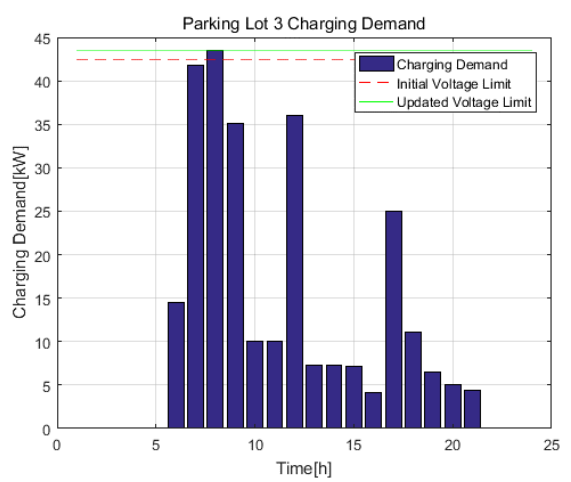

(c)

Figure 13. Integrated Charging Load of Parking Lot with MPC (a) PL1, (b) PL2, (c) PL3.

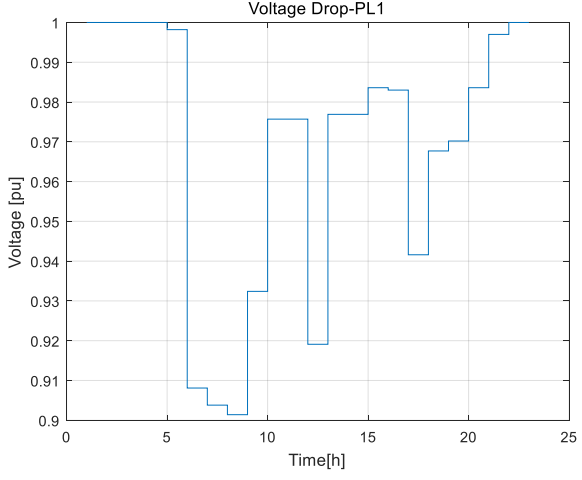

(a)

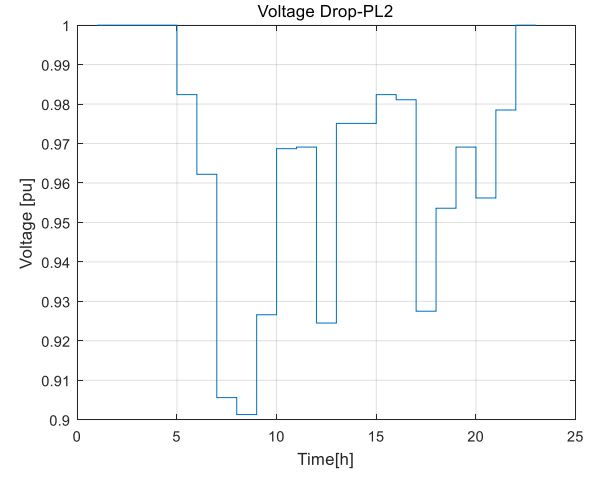

(b)

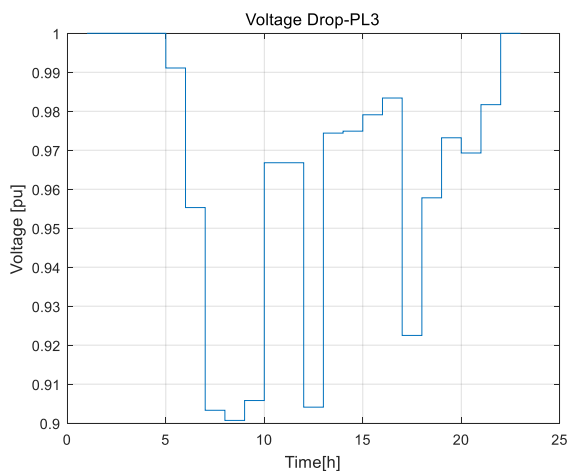

(c)

Figure 14. Parking Lot Voltage Drop with MPC (a) PL1, (b) PL2, (c) PL3. 
In Figure 13, the red and green lines show that the upper limit can be changed by the aggregator according to the conditions for that time. The red line indicates the charge upper limit generated when an initial bus voltage drop is expected. If the aggregator calculates the voltage variation value every hour, taking into account the local load at that time, and updates the constraint accordingly, the red line can be changed to a green line. In other words, the green line indicates the constraint modified by the aggregator for that time.

In the MPC framework, the upper limit for charging in parking lots 1 and 2 is reduced by the aggregator compared to the initially set value. This result means that a tighter constraint is created because more voltage variations are expected than those initially expected at that time. On the other hand, in the case of parking lot 3, the updated upper limit is relaxed compared to the initial value. Since the purpose of EV charging is to minimize the cost, if the voltage variation is within an acceptable range, the constraint is relaxed to charge as much as possible at the time the electric price is low.

At other times, the same strategy is applied, but since the creation of additional constraints is not necessary, the charging schedule is determined by only taking into account the minimization of the charging rate. As mentioned earlier, due to the constraints applied at 7:00 and 8:00, charging is limited at that time. Therefore, it can be confirmed that the charging is performed at a higher electric price in order to satisfy the condition for the required charging amount during the time when the EVs are parked in the parking lot. As shown in Figure 14, when optimum charging is performed considering the voltage limit, it is confirmed that the voltage variation is always less than $10 \%$.

\section{Conclusions}

This paper proposes an optimal charging strategy for EVs considering the voltage stability of the power system by using an MPC-based framework in the EV parking lot. The EV parking lot is stochastically modeled by considering the uncertain characteristics of the EVs, the voltage limit of the power system is analyzed in real time through the MPC framework, and the optimal charging limit is set to prevent voltage variation. The aggregator identifies and sets the charging limit in real time according to which the EV can minimize the impact on the power system while charging at a minimum cost. The proposed charging strategy is verified using MATLAB/Simulink based on the IEEE 15 bus system with EV parking lots. When the voltage drop violating the condition for voltage variation of the power system is not expected, the charging is implemented for minimizing the charging cost considering only the electric price of the charging. As a result, when only the charging rate is considered, many EVs are charged at an inexpensive time, resulting in an overload and severe voltage variation. However, when the proposed strategy is applied, a voltage variation beyond the limit does not always occur.

In the future, it is necessary to study the EV charging scheme and V2G operation plan in a power system such as the DC microgrid, where there are various distributed energy sources including the renewable energy sources such as wind and solar power.

Author Contributions: B.-R.C. carried out the main research tasks and wrote the full manuscript, and W.-P.L. provided technical support to verify the proposed strategy in simulation software. D.-J.W. validated and double-checked the proposed strategy, the results, and the whole manuscript.

Funding: This research received no external funding.

Acknowledgments: This work is supported by the Korea Institute of Energy Technology Evaluation and Planning (KETEP) and the Ministry of Trade, Industry \& Energy (MOTIE) of the Republic of Korea (No. 20161210200610) and was supported by Basic Science Research Program through the National Research Foundation of Korea (NRF), funded by the Ministry of Education, Science and Technology (Grant no. NRF-2016R1D1A1B03935712).

Conflicts of Interest: The authors declare no conflict of interest.

\section{References}

1. Di Giorgio, A.; Liberati, F.; Canale, S. Electric vehicles charging control in a smart grid: A model predictive control approach. Control Eng. Pract. 2014, 22, 147-162. [CrossRef] 
2. Deilami, S.; Masoum, A.; Moses, P.; Masoum, M. Real-time coordination of plug-in electric vehicle charging in smart grids to minimize power losses and improve voltage profile. IEEE Trans. Smart Grid 2011, 2, $456-467$. [CrossRef]

3. Liberati, F.; Mercurio, A.; Zuccaro, L.; Tortorelli, A.; Giorgio, A.D. Electric Vehicles Charging Load Reprofiling. In Proceedings of the Mediterranean Conference on Control and Automation, Palermo, Italy, 16-19 June 2014.

4. Richardson, P.; Flynn, D.; Keane, A. Impact Assessment of Varying Penetrations of Electric Vehicles on Low Voltage Distribution Systems. In Proceedings of the IEEE Power and Energy Society General Meeting, Providence, RI, USA, 25-29 July 2010.

5. Bansal, S.; Zeilinger, M.N.; Tomlin, C.J. Plug-and-Play Model Predictive Control for Electric Vehicle Charging and Voltage Control in Smart Grids. In Proceedings of the IEEE Conference on Decision and Control, Los Angeles, CA, USA, 15-17 December 2014.

6. Liu, Z.; Wu, Q.; Oren, S.S.; Huang, S.; Li, R.; Cheng, L. Distribution Locational Marginal Pricing for Optimal Electric Vehicle Charging Through Chance Constrained Mixed-Integer Programming. IEEE Trans. Smart Grid 2018, 9, 644-654. [CrossRef]

7. Leou, R.C.; Su, C.L.; Lu, C.N. Stochastic Analyses of Electric Vehicle Charging Impacts on Distribution Network. IEEE Trans. Power Syst. 2014, 29, 1055-1063. [CrossRef]

8. Rezaee, S.; Farjah, E.; Khorramdel, B. Probabilistic Analysis of Plug-In Electric Vehicles Impact on Electrical Grid through Homes and Parking Lots. IEEE Trans. Sustain. Energy 2013, 4, 1024-1033. [CrossRef]

9. Shao, C.; Wang, X.; Wang, X.; Du, C.; Wang, B. Hierarchical Charge Control of Large Populations of EVs. IEEE Trans. Smart Grid 2016, 7, 1147-1155. [CrossRef]

10. Shao, C.; Wang, X.; Shahidehpour, M.; Wang, X.; Wang, B. Partial Decomposition for Distributed Electric Vehicle Charging Control Considering Electric Power Grid Congestion. IEEE Trans. Smart Grid 2017, 8, 75-83. [CrossRef]

11. Mirzaei, M.J.; Kazemi, A.; Homaee, O. A Probabilistic Approach to Determine Optimal Capacity and Location of Electric Vehicles Parking Lots in Distribution Networks. IEEE Trans. Ind. Inform. 2016, 12, 1963-1972. [CrossRef]

12. Lu, Z.; Qi, J.; Zhang, J.; He, L.; Zhao, H. Modelling dynamic demand response for plug-in hybrid electric vehicles based on real-time charging price. IET Gener. Transm. Distrib. 2017, 11, 228-235. [CrossRef]

13. Tan, J.; Wang, L. Real-Time Charging Navigation of Electric Vehicles to Fast Charging Stations: A Hierarchical Game Approach. IEEE Trans. Smart Grid 2017, 8, 846-856. [CrossRef]

14. Zhang, L.; Li, Y. Optimal Management for Parking Lot Electric Vehicle Charging by Two-Stage Approximate Dynamic Programming. IEEE Trans. Smart Grid 2017, 8, 1722-1730. [CrossRef]

15. Neyestani, N.; Damavandi, M.Y.; Shafie-khah, M.; Bakirtzis, A.G.; Catalão, J.P.S. Plug-In Electric Vehicles Parking Lot Equilibria With Energy and Reserve Markets. IEEE Trans. Power Syst. 2017, 32, 2001-2016. [CrossRef]

16. Carnacho, E.F.; Bordons, C. Model Predictive Control; Springer: Berlin/Heidelberg, Germany; New York, NY, USA, 1999.

17. Luo, X.; Chan, K.W. Real-time scheduling of electric vehicles charging in low-voltage residential distribution systems to minimise power losses and improve voltage profile. IET Gener. Transm. Distrib. 2014, 8, 516-529. [CrossRef]

(C) 2018 by the authors. Licensee MDPI, Basel, Switzerland. This article is an open access article distributed under the terms and conditions of the Creative Commons Attribution (CC BY) license (http://creativecommons.org/licenses/by/4.0/). 